\title{
UTILIZING ACTIVE KNOWLEDGE MODELS IN AN INFRASTRUCTURE FOR VIRTUAL ENTERPRISES
}

\author{
Frank Lillehagen, Einar Dehli, Lasse Fjeld \\ Computas AS, NORWAY, fli@computas.com
}

John Krogstie, Håvard Dingstad Jørgensen SINTEF, NORWAY,(jok,hdj)@informatics.sintef.no

\begin{abstract}
We present in this paper a novel approach to support the development and operations of virtual enterprises. The approach is based on utilizing active knowledge models (AKMTM). An AKM is a visual model of enterprise aspects that can be viewed, traversed, analyzed, simulated, adapted and executed by industrial users. The AKM-approach is currently applied in the EXTERNALproject (IST 1999-10091), where experiences from three comprehensive casestudies are used as a basis for the further development of the approach.

A major result of the project is the identification and development of a fourlayered infrastructure to support virtual enterprises. The four layers are: - the ICT layer, the knowledge representation layer, the work and model management layer, and the work performance layer.
\end{abstract}

\section{INTRODUCTION}

The business environment is getting increasingly more dynamic. Co-operation across traditional organizational boundaries is increasing, as outsourcing and electronic business is enabled by the Internet and IS in general. When such cooperation moves beyond the buying and selling of goods and well-defined services, there is a need for a flexible infrastructure that supports not only information exchange, but also knowledge creation and sharing.

An extended enterprise (EE) (also often termed virtual enterprise) is defined as a dynamic networked organization, being developed ad-hoc to reach a certain goal based on the resources of several existing co-operating enterprises. The EE-partners often comes from different countries, using different languages and having different cultural background. The EE-partners wants to harvest knowledge from the EE to be reused in the originating organization, or in other EE's.

The approach and working environments to enable this must allow dynamic, concurrent execution, modeling, and meta-modeling of solutions in distributed teams. This demands a new approach to enterprise integration and system engineering, which will be presented in this paper. We first present the concepts of Active Knowledge Models (AKM). We then present briefly the EXTERNAL ISTproject (EXTERNAL,1999), and the 4 layers of infrastructure that is provided through this project to make AKM-thinking a practical reality. Major aspects of the

The original version of this chapter was revised: The copyright line was incorrect. This has been corrected. The Erratum to this chapter is available at DOI: 10.1007/978-0-387-35585-6_68 
infrastructure is presented, before briefly positioning the work in context with related work as part of the concluding remarks.

\section{AKM THINKING AND THE EXTERNAL PROJECT}

An Active Knowledge Model is a visual externalization of enterprise aspects that can be operated on (viewed, traversed, analyzed, simulated, adapted and executed) by industrial users. What does it mean that the model is active? First of all, the visual model must be available to the users of the underlying information system at runtime. Second, the model must manage and drive the behavior of the computerized support-system. Third, the model must be dynamic, users must be supported in changing the model to fit their local reality, enabling tailoring of the system's behavior.

The knowledge representation must be adapted to the type of medium, the language for encoding and the capabilities of the carrier. The language for encoding can span from icons and natural language to state-sensitive layered patterns. The medium varies from paper to the WWW. Knowledge encoding can either be performed using natural language, visual patterns, or views of described artifacts with embedded methods. Currently we are engaged in developing graphical patterns to improve our visual communication of situated knowledge.

\subsection{The AKM Approach}

AKMs of Enterprises imply that the enterprise is extended by distributed teamworking on layers of knowledge, and that modeling, meta-modeling and work process can be performed in parallel. The AKM technology redefines what we mean by a model by introducing the characteristics of knowledge as a fundamental aspect in EE engineering solutions. Knowledge creation and management are active capabilities in work process execution.

AKM implementation is dependent on a rich generic knowledge base and powerful development and extension capabilities of the $\mathrm{EE}$ infrastructure. Being able to support collaborative work and managing knowledge will decide the quality of the solution. The usage and value of the solution also depend on the competence and knowledge of the teams involved. The starting point for EE Engineering projects in our experience often is that industries have formed competent teams for Project Management, Knowledge Management, EE Engineering, EE Operation, and Solution Support. The first major activity among these teams, is to detail and agree on purpose and scope of the EE to be developed, then to start capturing knowledge by gathering points of view and relevant information. The modeling environment is used for this knowledge mapping, analyses, and deliberation among teams.

\subsection{Overview of the EXTERNAL project}

In the EXTERNAL project IST 1999-10091 we are working to develop a technical and conceptual infrastructure to support the AKM approach.

The most innovative contributions from EXTERNAL can be summarized as: 
- Implementing EEs based on AKM technology

- Implementing the multiple views of active objects, exploiting the reflective, recursive, repetitive and replicable nature of (situated) work process knowledge.

- Applying the model evolution and management processes.

- Implementing a four-layered infrastructure with open EE formation, engineering and operation capabilities and architectures for dynamic IT component inclusion, knowledge representation, work and model management and dynamic user environment generation.

- Implementing an EE Engineering, management and operations methodology supported by the layered Infrastructure.

The infrastructure (Karlsen, 2001), methodology (Krogstie, 2000), case-study solutions, and the EXTERNAL project itself are developed in parallel. The infrastructure will support and implement the methodology, provide project management services, and implement work processes and services for engineering customer EE solutions. We will in the next section describe the four-layered infrastructure in more detail.

\section{LAYERS OF INFRASTRUCTURE - MAKING AKM A REALITY}

The EE infrastructure is best described as consisting of four layers. These layers are identified as:

- Layer 1, the ICT layer: - defining and describing the ICT platform, the software architectures, tools, software component, connectivity and communications.

- Layer 2, the Knowledge Representation layer: - defining and describing constructs for modeling, developing, sharing and managing the contents of model and meta-model repositories.

- Layer 3, the Model and Work Management layer; - modeling the customer solution, adapting engineering processes, and implementing work processes.

- Layer 4, the Work Performance layer; - implementing customer solutions, generating work environments as personalized and context-sensitive GUI's (worktops) available through portals, and performing work.

\subsection{The ICT layer}

The current version of the infrastructure is an integration of the enterprise and process modeling-tools brought into the EXTERNAL project by the partners. The following software tools are the key components of the current ICT layer.

- METIS (Lillehagen, 1999), a general purpose enterprise modeling and visualization tool,

- XCHIPS (Haake and Wang, 1997), a co-operative hypermedia tool integrated with process support and synchronous collaboration,

- Vité (Kuntz, 1998) (now called SimVision), a project simulator used to analyze resource allocation, highlighting potential sources of delay and backlogs. 
- WORKWARE (Jørgensen and Carlsen, 1999), an emergent workflow management system with to-do-lists, document sharing, process enactment and awareness mechanisms.

Figure 1 depicts the main parts of the upcoming version. The architecture has 3tiers, clients, application servers, and data servers, utilizing web services i.e. a server application communicating with its clients solely through standard web protocols such as HTTP and exchanging data through XML.

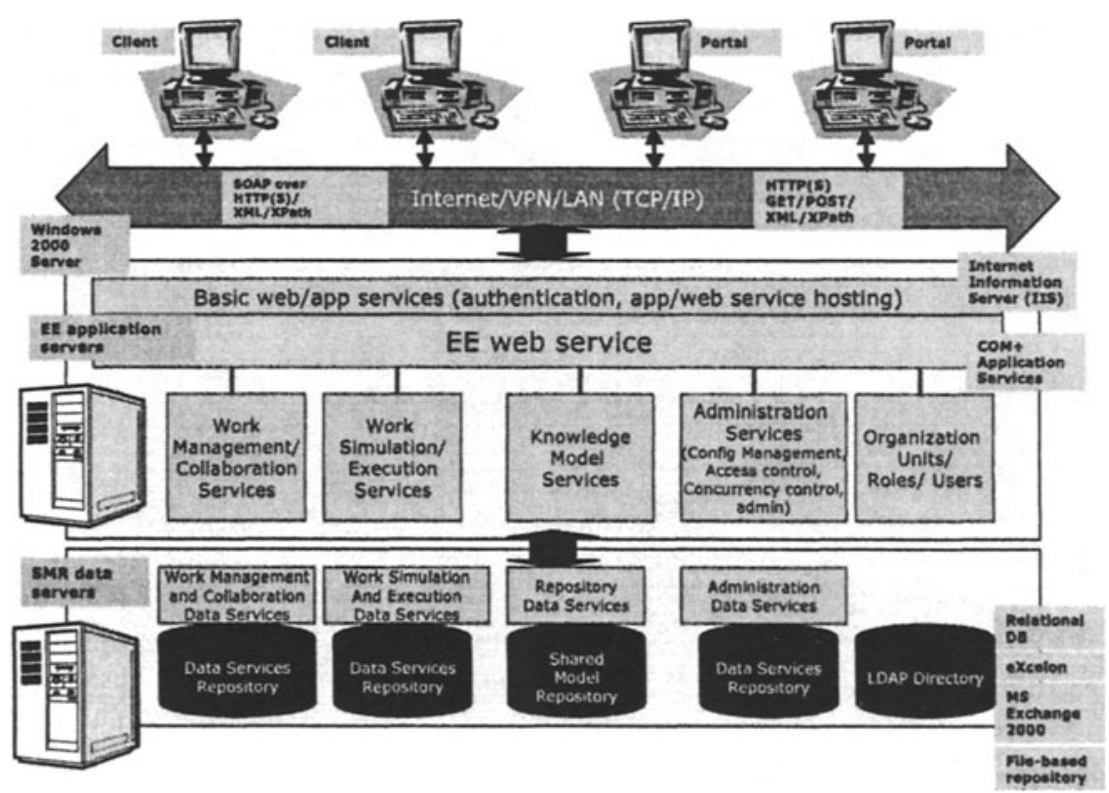

Figure 1: The architecture and components of the EE Infrastructure, ICT layer.

\subsection{The knowledge representation layer}

The Knowledge Representation layer defines how models, meta-models and metadata are represented, used and managed. METIS is used to manage models, modeling languages and meta-data. The model content can be persistently stored in the shared model repository. Upcoming versions will support project, team and work administrative processes and an administration database. Model contents, metamodel versions, revisions and variants, and meta-data hierarchies that are local, project specific or global will be separately managed. The architecture involves work processes that manage the project administration database (organization, roles, users) and the meta-model repository, and that save accumulated experiences and life-histories for change and configuration management and situated learning purposes. 


\subsection{The model and work management layer}

Model and work management will model and implement repetitive work processes for the engineering processes, and provide services to support the EE teams. A portal-based solution host the worktops which is used to do project and model management, including management of and access to the repository. The work management services of the current version are typically built using METIS and performance is supported using WORKWARE, were updates to the model can also be done interleaved with model execution.

In versions 1.5 and 2.0 we will model and implement work processes as active, reflective objects where appropriate. Model and work management will therefore be implemented as immersed, rule driven and reflective work processes. The architecture of this layer is the management rules embedded in use case work processes, the model engineering work processes, and the life-cycle management model automatically creating life-history, when teams are executing work processes.

\subsection{The work performance layer}

The portal-based solution is used to register and qualify users, and all knowledge and information is accessed using the various tools. As part of WORKWARE the modeled tasks are typically also executed through the invocation of tools and applications. The focus in WORKWARE is to set up the right context for a certain task and to identify the resources needed to do the task. The actual execution is done by invoking the appropriate tool or application.

In future versions, user environments will be generated as designed by performing specific engineering work processes. The EE Infrastructure, version 2.0, will also be able to support the actual work execution, executing active objects, allowing us to model the actions and then to perform them accordingly. Individual and collaborative knowledge work, security features such as electronic signatures, and simulation and role-play for work driven training will be supported.

Users will typically access their solutions through project portals. Any EE project portal user environment must have support for methodology adaptation, project working, and for communications, co-ordination and collaboration among teams and groups. Project work management, reporting and other services will have to be supported, and finally project work execution must be performed with possibilities for repetition and management services, providing safety, security and privacy to knowledge workers.

The layers of the user environment are illustrated in the figure below. The project-top will support the project management team. The contents may be an overview of the project, adopted management principles, applicable methodologies, project work-break-down structure, results, plans and tasks, technologies and resources, and status reporting and calculations.

The work and model management worktop contains an overview of tasks and status of work being processed. The tasks can be viewed in at least four ways: indented lists, tree-structures, control-panels and task-plans. The status can be communicated by the use of colors.

The work execution worktop is generated from the work process description for each task. The generation happens automatically, when task status is set to 
execution. The generation should take into account the skill level of the allocated team and personnel

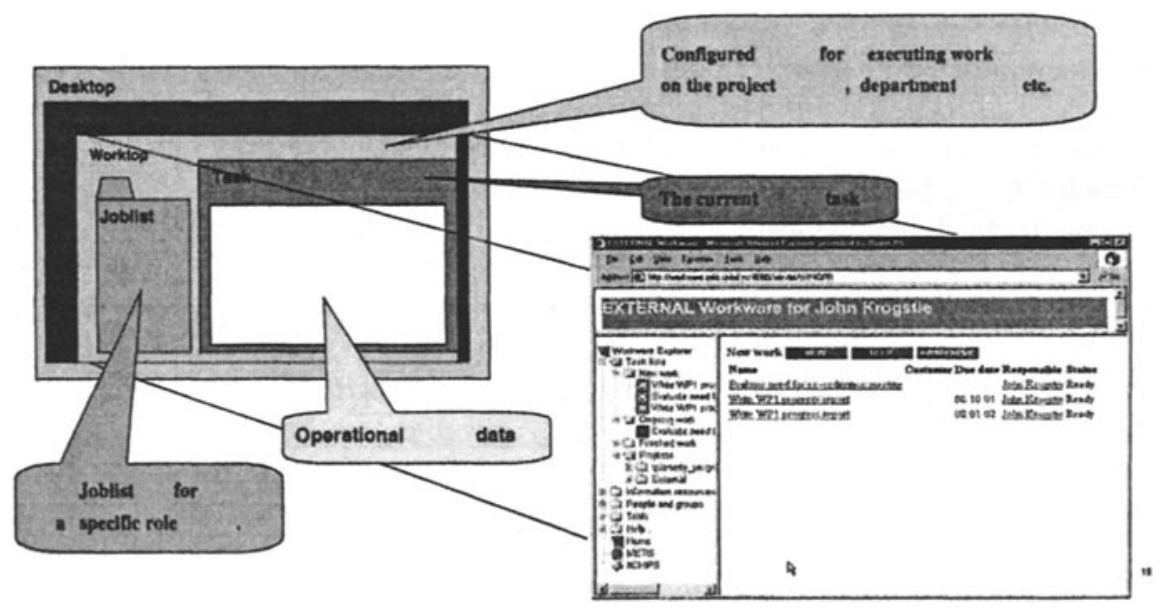

Figure 2 - The layers of dynamic User Environments.

Work management is currently supported through the concept of work-lists. A worklist consists of a set of tasks to be performed with priorities, due dates etc. A task represents a piece of work that needs to be done. The work management is based on a process representation in a form that supports automated manipulations, such as modeling, or enactment by a workflow engine. The process definition consists of a network of activities and their relationships, criteria and rules to indicate the start and termination of the process, and information about the individual activities, such as participants, associated IT applications and data. The EE Modeling Language developed in the project is the first step towards such a representation. The process definition will be extended with rules for more explicit handling of conditions to control start, execution and termination. The collaboration service will be a server application that provides support for synchronous and asynchronous work collaboration. Synchronous collaboration will be supported either through the use of NetMeeting, WebEx, or XCHIPS/Dyce.

\subsection{Work Simulation/ Execution Services}

The work simulation services are server applications that provide support for different kinds of simulation of the EE models. An example of such a service is the kind of simulation SimVision provides to simulate project loads. The work execution service is based on the same process model as defined in the work management service only it is extended with language constructs to specify actions in addition to conditions. 
Actions are the atomic units of execution inside tasks. An action can be a call into an object model, realized as an object expression or any other means of making something happen in the computer system on which the process is executed. Conditions are used to determine when a process step can be executed, when execution of the step is completed, and to choose between different actions in the task. Each process instance represents a separate thread of execution of the process, which may be controlled independently and will have its own internal state and externally visible identity that may be used as a handle, for example, to record or retrieve audit data relating to the individual enactment. (Karlsen, 2001; Larsen, 1999).

\section{RELATED WORK}

The EXTERNAL infrastructure combines a number of mechanisms that use active models for customizing and tailoring the information systems, e.g. simulation, workflow enactment, awareness, user interface layout and navigation design.

With respect to supporting dynamically networked organizations, most B2B Ebusiness frameworks (Shim, 2000) focus on information exchange and business transactions. This is also the case with newer frameworks such as ebXML. They lack support for the dynamic and knowledge-intensive parts of inter-organizational processes.

Enterprise ontologies have been proposed as a way of solving the communication problems arising from different interpretative frameworks in different organizations (Fox and Gruninger, 1997). This approach is based on conventional notions of model interpretation, i.e. the Turing paradigm, where the technical actor interpretation is fully automated and no interaction is allowed to aid interpretation, and not the more powerful interaction machine paradigm (Jørgensen, 2001; Wegner, 1997). The main characteristic of an interaction machine is that it can pose questions to human actors (users) during its computation. The problem solving process is no longer just a user providing input to the machine which then processes the request and provides an answer (output), it is a multi-step conversation between the user and the machine, each being able to take the initiative. Active models are also directed towards ongoing modeling, model interpretation, and activation by the end users.

Workflow management systems have also been proposed as a solution for interorganizational collaboration (van der Aalst and Weske, 2001; Casati, 2000; Reichert, 1999). The focus of EXTERNAL on knowledge intensive processes requires a degree of flexibility not enabled by conventional production workflow systems.

Another popular solution for cross-enterprise integration is middleware frameworks like OMG's CORBA. The recent shift in the focus of OMG to modeling (MDA and UML), standardization of meta-object integration, business object frameworks and workflow management facilities indicate an interest in modeldriven enterprise integration also from the more technical side. Whether these software engineering approaches are directly transferable to active modeling remains to be investigated thoroughly. 


\section{CONCLUSION AND FURTHER WORK}

The current version of the EXTERNAL infrastructure is in use in the three EXTERNAL case studies, and parts of it are also used in other, commercial projects. It has been found adequate for building the case-study models for use in the project. It also demonstrates the power and possibilities of the AKM technology in finding solutions to the industrial challenges mentioned in the introduction. However, the current version has many shortcomings when it comes to supporting distributed project team-working for EE creation and operation in an industrial setting. Work on version 1.5 is well underway and will introduce worktops for project management, new meta-modeling capabilities, improved repository services, and process support for model use and management.

\section{REFERENCES}

1. van der Aalst, W. M. P. and Weske, M The P2P Approach to Inter-organisational Workflows in in Advanced Information Systems Engineering - Proceedings of CAiSE 2001, Dittrich, K. R., Geppert, A. and Norrie, M. C. (Eds.) pp 140-156, Interlaken, Switzerland, 4-8 June 2001.

2. EXTERNAL Consortium 'EXTERNAL - Extended Enterprise Engineering, Resources, Networks and Learning, EU IST-project, IST-1999-10091.

3. Casati, F., Innicki, S., Jin, L., Krishnamoorthy, V. and Shan, M.-C. Adaptive and Dynamic Service Composition in eFlow, in Advanced Information Systems Engineering - Proceedings of CAiSE 2000, vol. LNCS 1789, B. Wangler and L. Bergman, Eds. Stockholm, Sweden: Springer, 2000.

4. Fox, M. S. and Gruninger, M. On Ontologies and Enterprise Modelling, International Conference on Enterprise Integration Modelling Technology 97, 1997.

5. Haake, J. M. and Wang, W. Flexible Support for Business Processes: Extending Cooperative Hypermedia with Process Support, GROUP '97, Phoenix, Arizona USA, 1997.

6. Jørgensen, H. D. and Carlsen, S. Emergent Workflow: Integrated Planning and Performance of Process Instances, Workflow Management '99, Münster, Germany, 1999.

7. Jørgensen, H., "Interaction as a Framework for Flexible Workflow Modelling", GROUP 2001, Boulder, Colorado, October 2001.

8. Karlsen, D. et al, "D3 - EE Infrastructure , version 1.0," A deliverable(D3) from the EXTERNAL project, Lysaker, March 2001, and other publications on EE / VO infrastructures.

9. Krogstie J. et al, "D2 - EE Methodology , version 1.0," A deliverable(D2) from the EXTERNAL project, Lysaker, Dec. 2000, and other publications on EE/ VO methodologies.

10. Kuntz, J. C., Christiansen, T. R., Cohen, G. P., Jin, Y. and Levitt, R. E. The Virtual Design Team: A Computational Simulation Model of Project Organizations, Communications of the ACM, vol. 41, no. 11 , 1998.

11. Larsen, Lars Bjørn et al, "The Concepts of the Globeman 21 project", Globeman21 report D1.1 and presentations, DTU, Copenhagen, June 1999.

12. Lillehagen, F. Visual Extended Enterprise Engineering Embedding Knowledge Management, Systems Engineering and Work Execution, IEMC '99, IFIP International Enterprise Modelling Conference, Verdal, Norway, 1999.

13. Reichert, M., Bauer, T. and Dadam, P. Enterprise-Wide and Cross-Enterprise WorkflowManagement: Challenges and Research Issues for Adaptive Workflows, Enterprise-wide and Crossenterprise Workflow Management, Paderborn, Germany,, 1999.

14. Shim, S. S. Y., Pendyala, V. S., Sundaram, M. and Gao, J. Z. Business-to-Business E-Commerce Frameworks, IEEE Computer, vol. 33, no. 10, 2000.

15. Wegner, $\mathrm{P}$. Why interaction is more powerful than algorithms, Communications of the ACM, vol. 40, no. 5, 1997. 